\title{
Adaptivity and Safety in Location-Based Games
}

\author{
João Jacob \\ FEUP \\ Rua Dr. Roberto Frias, 4200-465 \\ Porto, Portugal
}

\author{
Rui Nóbrega, António Coelho, Rui Rodrigues \\ INESC TEC and FEUP \\ Rua Dr. Roberto Frias, 4200-465 \\ Porto, Portugal
}

\begin{abstract}
Location-based games require, among other things, obtaining or computing information regarding the players' physical activity and real-world context. Additionally, ensuring that the players are assigned challenges that are adequate and safe for the current context (both physical and spatial) is also important, as it can improve both the gaming experience and the outcomes of the exercise. However, the impact adaptivity has in the specific case of location-based exergames still has not been researched in depth. In this paper, we present a location-based exergame capable of adapting its mechanics to the current context.
\end{abstract}

Keywords-exergames, location-based games, player adaptivity, game design, mobile computing

\section{INTRODUCTION}

Location-based games (games that use the player's physical location and incorporate it into the game in some way [1]) require obtaining or computing information regarding the players' physical activity, current location and context. Additionally, ensuring that the players are assigned challenges that are adequate to their physical ability, safe and adapted for the current context (both physical and spatial) is also important, as it can improve both the gaming experience and the outcomes of the game. So, the relevant information gathering of both player and context proves to be useful in determining the performance of the former in the latter. There are some models that combine the challenge's difficulty and the player's skill, for both regular games and, more recently, exergames: the Game Flow Model [2] or the Dual Flow Model [3] respectively. The work described in this paper presents techniques for using sources of geographical information to provide location-based games with context-aware content.

\section{A. Related Work}

Despite being recent, location-based games have already become popular [4]. These games have since then gained a considerable amount of popularity. For instance, Geocaching [5] is one such popular location-based game, with over one million of users. Games such as "Zombies, Run!" [6], a jogging-whileescaping-from-zombies exergame, or "Geo Wars" [7] a location-based strategy exergame, which promotes and rewards the player for performing physical activity in his/her surrounding environments. More recently, Ingress [8] and Pokémon Go [9], both social and location-based games with some augmented reality capabilities have also been relatively successful.

The importance of game adaptivity in location-based games and exergames was also explained by Jacob et al. [10], as a means to overcome the issues these types of games commonly present. These games were defined by D.Charles and B. Magerko as games that recognize player input and are able to adequately change their behavior (be it gameplay mechanics or game elements [11],[12]) so as to ensure a better experience. Adaptivity plays an important role in this work as it will enable the game to dynamically change itself to better accommodate the current player's status.

\section{GhostChase}

GhostChase, is a mobile location-based exergame that showcases the combination of the GeoStream [13] platform (see Fig. 1) with a game adaptivity framework. In it, a set of ghosts is chasing the player as if they were in the surroundings of the real-world location of the player. The player must therefore flee from the pursuing ghosts by physically moving in the real world, reaching a game-suggested haven (a real physical location) before they catch him/her. The game is played on an Android smartphone with both headphones and optionally an Android Wear enabled smartwatch.

Once the game starts, the player can see (Fig. 2) and hear the game. The player is represented by the red dot at the center of the screen. White dots represent the position of ghosts and the purple line is the recommended path to the safehouse. Additional information (such as calories' burn rate, volume of oxygen consumed and other) is conveyed in the lower-left corner of the screen.

Some of this information, such as speed and heart rate, are values read directly from the devices' sensors or via previous user input, in the case of weight.

The Relative Gross Volume of Oxygen consumed and the player's Burn Rate are computed using the following formulae, already studied [14]:

$$
\begin{gathered}
\text { RGVO2 }\left(\frac{\frac{m L}{K g}}{\min }\right)=0.2 * \text { Speed }+0.9 * \text { Speed } * \text { Slope }+3.5 \\
\text { Burn Rate }\left(\frac{\text { Cal }}{\min }\right)=\frac{R G V O 2}{1000} * \text { Weight } * 5
\end{gathered}
$$

Note that the above equations will over-estimate oxygen consumption [14] and burn rate.

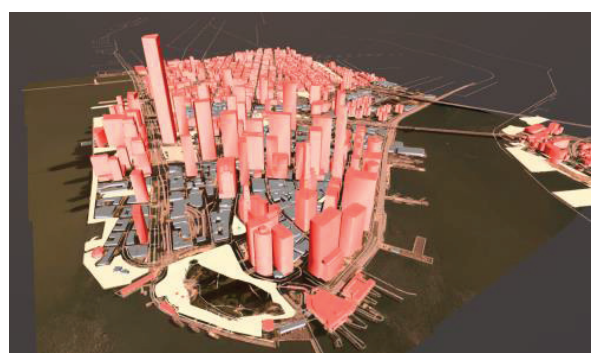

Fig. 1- Island of Manhattan (via GeoStream) 


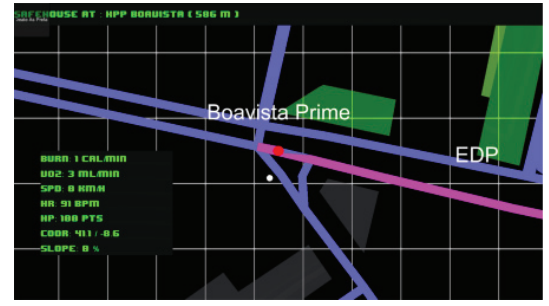

Fig. 2- GhostChase Appearance

Since the game requires the player to run around, it encourages only glancing the phone when absolutely needed, as it guides the player with audio-based turn-by-turn navigation to the safehouse. Whenever ghosts are getting closer to the player, a continuous beeping sound is played. The pitch, tempo and volume of that sound increase whenever the ghost gets closer to the player. Conversely, they fade into silence when the player evades the ghost(s). If a ghost touches the player, it will make the player lose health at the rate of one health point per second. If the player's health reaches zero, the game is lost. If the player safely reaches the safehouse, the game is won.

GhostChase makes use of GeoStream to generate the buildings' footprints, calculate the safehouse position (a hospital in the area, if none exists, the game will only spawn ghosts and effectively start when the player's surroundings contain one) and determine the shortest known path to it. It also makes use of several local sensors to determine the player's real-world position, speed and heart rate. It is noteworthy that other sources of information are supported by GeoStream and could be used to foster adaptivity and safety, such as time of day, weather conditions, type of road, real time traffic information among others. For instance, the safehouse could just as easily be another type of point of interest, with no changes in the game's mechanics.

To test how adaptivity and player safety in location-based games can be achieved, two profiles for the game were created: GhostChaseB - a basic, non-adaptive version of the game. Ghosts spawn at a fixed rate, every 30 seconds, approximately 50 meters behind the player - and GhostChase $\mathrm{A}$ - an adaptive version of the game. Using GeoStream, the game has access to context-sensitive geographical information. This version of the game stops ghosts from spawning when the player is within 50 meters of a crosswalk for safety reasons. Simulating three distinct locations with mock GPS input to ascertain if there are differences between these two versions suggests that GhostChaseA is indeed capable of being aware of nearby
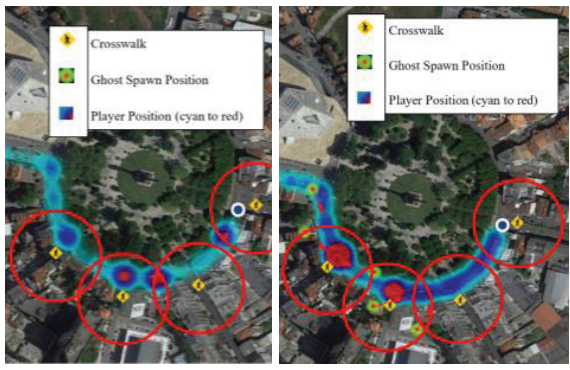

Fig. 3- Results in GhostChaseA vs GhostChaseB crossroads (if this information is available via GeoStream) and does change the spawning mechanics as designed, whereas GhostChaseB doesn't.

\section{CONCLUSIONS AND FUTURE WORK}

We have presented GhostChase, a location-based game that is able to access varied geo-information sources and incorporate that information in-game thanks to GeoStream, an API for aggregating and accessing geo-referenced data from several third-party sources.

Our preliminary results with GhostChase and GeoStream show a potential benefit for the player's experience and safety in making location-based games aware of potentially dangerous real-world situations and adapting their mechanics accordingly. It is our intention to test this game with users on different locations and later provide a generic methodology through which already existing location-based games may adapt their own challenges and mechanics in accordance with contextsensitive information.

\section{REFERENCES}

[1] O. Sotamaa, “All The World's A Botfighter Stage: Notes on Location-based Multi-User Gaming," Cultures, pp. 35-44, 2002.

[2] P. Sweetser and P. Wyeth, "GameFlow: a model for evaluating player enjoyment in games," Comput. Entertain., vol. 3, no. 3, p. 3, 2005.

[3] J. Sinclair, P. Hingston, and M. Masek, "Exergame development using the dual flow model," 5th Int. Conf. Intell. Environ. - IE'09, pp. 1-7, 2009.

[4] S. Matyas, "Playful Geospatial Data Acquisition by Location-based Gaming Communities," Int. J. Virtual Real., vol. 6, no. 3, pp. 1-10, 2007.

[5] K. O’Hara, "Understanding geocaching practices and motivations," Proc. SIGCHI Conf. Hum. ..., p. 1177, 2008.

[6] K. Erenli, "The impact of gamification: A recommendation of scenarios for education,” Interact. Collab. Learn. (ICL), 2012 15th Int. Conf., pp. 18, 2012.

[7] J. T. P. N. Jacob and A. F. Coelho, "Geo Wars-The development of a location-based game,” Rev. Prism. Com, no. 14, pp. 1-13, 2011.

[8] H. Hodson, "Google's Ingress game is a gold mine for augmented reality," New Sci., vol. 216, no. 2893, p. 19, Dec. 2012.

[9] A. Colley et al., "The Geography of Pokémon GO: Beneficial and Problematic Effects on Places and Movement," in CHI 2017, 2017, pp. 1179-1192.

[10] J. Jacob and A. Coelho, "Issues in the Development of Location-Based Games,” Int. J. Comput. Games Technol., vol. 2011, pp. 1-7, 2011.

[11]F. Berger, "Evaluating an Implementation of an Adaptive Game-Based Learning Architecture," User Model. Adapt. Pers., vol. 7379, no. January, 2012.

[12] R. Lopes and R. Bidarra, "Adaptivity Challenges in Games and Simulations: A Survey,” IEEE Trans. Comput. Intell. AI Games, vol. 3, no. 2, pp. 85-99, Jun. 2011.

[13] J. S. V Gonçalves, J. Jacob, R. J. F. Rossetti, A. Coelho, and R. Rodrigues, "An integrated framework for mobile-based ADAS simulation," Lect. Notes Control Inf. Sci., vol. 13, pp. 171-186, 2015.

[14] A. RUIZ and N. SHERMAN, "An Evaluation of the Accuracy of the American College of Sports Medicine Metabolic Equation for Estimating the Oxygen Cost of Running," J. Strength Cond. Res., 1999. 